\title{
Itinerario formativo e investigativo en sistematización de experiencias (IFISE)
}

Formative and Research Itinerary in Systematization of Experiences

Itinerário de formação e pesquisa em sistematização de experiências

Diego Fernando Barragán-Giraldo* (iD) orcid.org/0000-0002-8270-118x María Luz Ceci lbarra-Mogollón ** (iD) orcid.org/0000-0002-1659-4179 Tito Hernando Pérez-Pérez ${ }^{* * *}$ (ID

Para citar este artículo: Barragán-Giraldo, D.F., Ibarra-Mogollón, M.L. y Pérez-Pérez, T. H. (202O). Itinerario formativo e investigativo en sistematización de experiencias (IFISE). Revista Colombiana de Educación, 1(79)., 243-272. https://doi.org/10.17227/rce.num79-6349

\section{(c) $(1) \Theta$}

* $\quad$ Doctor en Educación y sociedad. Investigador docente en la Universidad de La Salle, Bogotá, Colombia. Correo electró- nico: dibarragan@unisalle.edu.co

** Magistra en Tecnología Educativa. Profesora de la Secretaría de Educación Distrital de Bogotá, Colombia. Correo electrónico: mlibarra@educacionbogota.edu.co

*** Magíster en Docencia. Docente investigador de la Universidad de La Salle y de la Universidad Santo Tomás, Bogotá, Colombia. Correo electrónico: tperez@unisalle.edu.co 


\section{Resumen}

Este artículo presenta los desarrollos investigativos de dos tutores de investigación de una maestría en educación que, al orientar los procesos de formación desde la sistematización de experiencias, pudieron reconstruir su propia ruta formativa y proponer una estrategia para orientar este tipo de trabajos de nivel posgradual. En la introducción, se muestra el contexto general de política pública que permitió a los estudiantes su formación como magísteres. Luego, se establecen algunos enunciados sobre los conceptos de práctica, experiencia y sistematización de experiencias. Posteriormente, se establece la ruta metodológica desarrollada en el proceso investigativo: aprender con los otros, visitar la teoría, visitar los contextos, visitar la metodología, ajustar lo formal, socializar y generar teoría; la cual llevó a los autores a proponer el Itinerario formativo e investigativo en sistematización de experiencias (Ifise), que como estrategia formativa posibilitó desarrollar capacidades investigativas en los maestrantes. Al finalizar el texto se presentan los resultados, de los cuales, se destaca que en el proceso de formación se incentivó la apropiación del rol de investigador, por medio de la ruta metodológica planteada, de manera que se aportó a la configuración de las metodologías de sistematización de experiencias y se consolidaron comunidades de práctica.

\section{Palabras clave}

investigación cualitativa; prácticas educativas; educación popular; sistematización de experiencias

\section{Keywords}

qualitative research; educational practices; popular education; systematization of experiences

\begin{abstract}
This article presents the research developments of two research tutors from a master's degree in education who, by guiding the training processes from the systematization of experiences, were able to reconstruct their own training path and propose a strategy to guide this type of postgraduate level work. In the introduction the general context of public policy that allowed students to train as masters is shown. Then, some statements are made about the concepts of practice, experience and systematization of experiences. Subsequently, the methodological route developed in the investigative process is established: learning with others, visiting the theory, visiting the contexts, visiting the methodology, adjusting the formal, socializing and generating theory; which led the authors to propose the training and research itinerary in systematization of experiences, which as a training strategy made it possible to develop research skills in teachers. At the end of the text the results are presented, of which, it is highlighted that in the training process the appropriation of the role of researcher was encouraged, through the proposed methodological route, in a way that it contributed to the configuration of the methodologies of systematization of experiences and consolidated communities of practice.
\end{abstract}

\section{Resumo}

Este artigo apresenta os desenvolvimentos de pesquisa de dois tutores de mestrado em educação que, ao orientar os processos de formação a partir da sistematização de experiências, conseguiram reconstruir seu próprio caminho de formação e propor uma estratégia para orientar esse tipo de trabalho de pós-graduação. Na introdução, é mostrado o contexto geral das políticas públicas que permitiram que os alunos treinassem como mestrandos. Em seguida, são feitas algumas afirmações sobre os conceitos de prática, experiência e sistematização de experiências. Posteriormente, é estabelecido o caminho metodológico desenvolvido no processo investigativo: aprender com os outros, visitar a teoria, visitar os contextos, visitar a metodologia, ajustar a forma, socializar e gerar teoría; o que levou os autores a propor o itinerário de formação e pesquisa em sistematização de experiências, que como estratégia de formação possibilitou o desenvolvimento de habilidades de pesquisa em mestrandos. Ao final do texto, são apresentados os resultados, dos quais, destaca-se que no processo de formação a apropriação do papel de pesquisador foi incentivada, através da rota metodológica proposta, de forma que contribuísse para a configuração das metodologias de sistematização de experiências e se consolidaram comunidades de prática.

\section{Palavras-chave}

pesquisa qualitativa; práticas educativas; educação popular; sistematização de experiências 
He aquí

- dijo el maestro, enseñando los paquetes-

¡mis memorias!

Edmundo de Amicis, Corazón. Diario de un niño

\section{Introducción}

¿Para qué mejorar la educación? Las respuestas a esta pregunta parecen obvias; sin embargo, lo que en primera instancia es tan claro posee infinidad de rutas de acercamiento, precisamente por la complejidad del fenómeno educativo. Una de las respuestas tiene que ver, necesariamente, con la posibilidad de constituir un mundo mejor; claro que siempre está a la vista un interrogante derivado: ¿futuro mejor para quiénes? Este asunto de una u otra forma implica pensar sobre los fines de la educación: "la educación tiene que enfrentarse hoy, como en otros tiempos, a los problemas que plantea cada presente en el espacio concreto de la historia que lo determina" (Lledó, 2018, p. 13). La preocupación sobre el para qué formar es un asunto del orden político y la pregunta por el cómo remite a la reflexión educativa y pedagógica.

En este contexto, los diferentes países se han preocupado por fortalecer los aspectos concernientes a la educación, desde la política pública, fijando la mirada, entre otros aspectos, en el fortalecimiento de la formación de los docentes, quienes son actores de relevancia capital para elevar la calidad de la educación de las naciones: "la responsabilidad principal de los docentes es impartir una enseñanza de alta calidad" (Unesco, 2017, p. 75). Sin embargo, los docentes no pueden convertirse en el tema central para mejorar la calidad de la educación; la anhelada calidad está asociada a la inversión social, la equidad y, en general, a la manera como los Estados invierten en todo el sistema educativo y este sistema se articula a la sociedad misma. Lo anterior significa que aunque el profesor no es el único responsable de la mejora de la educación, sí es una figura importante y relevante en este proceso (Unesco, 2016).

Así, en muchos lugares del mundo la formación de profesores empieza a tomar relevancia, especialmente porque parece contribuir a la mejora de la calidad educativa de las naciones. Por ejemplo, la Unesco en sus sucesivos informes de seguimiento a la educación en el mundo hace múltiples llamados a fortalecer las competencias docentes para tener maestros cualificados en los diversos países; sin embargo, aparecen dos limitaciones: "en primer lugar, todavía queda mucho camino por recorrer antes de que se pueda elaborar una medición significativa del número de docentes capacitados [...] la segunda dificultad es la escasez de datos" 
(Unesco, 2017, p. 278). Estas limitaciones se refuerzan, según el informe 2017/8, debido a que "la desconexión que existe entre la formulación de la meta (que habla de 'cualificados') y del indicador mundial (que habla de 'formados') contribuye a la confusión acerca de qué significa ser un docente cualificado" (Unesco, 2017, p. 278).

A pesar de las limitaciones enunciadas y la confianza en la formación de los docentes expresada en los informes, estos ponen de manifiesto la necesidad de prestar atención a la educación como elemento importante de transformación social y, especialmente, a la formación docente: "si se quiere acabar con la crisis del aprendizaje, todos los países, ricos y pobres, han de procurar que todos los niños puedan tener acceso a un docente bien capacitado y motivado" (Unesco, 2014b, p. 340). En el documento se insiste en que no basta con tener suficientes docentes, sino que estos deben estar capacitados para ampliar reflexivamente sus estrategias pedagógicas (Unesco, 2014a). Ahora bien, si se cuenta con muchos profesores, pero algunos de ellos tienen una escasa formación, esto puede comprometer la calidad de la educación de muchos niños que Ilegan a la escuela: "los países con un alto número de docentes no capacitados se enfrentan a la doble tarea de contratar a docentes capacitados y de formar a los docentes no capacitados" (2014a, p. 9); así, se ratifica la necesidad la formación docente como ruta prioritaria para mejorar la educación. En el informe se insiste en que "no será suficiente con aumentar la cantidad de docentes; también es preciso mejorar la calidad con docentes bien formados y motivados" (Unesco, 2015, p. 229). En síntesis: motivación, perfeccionamiento profesional permanente y mejores salarios para evitar que los buenos maestros salgan de las escuelas son algunas de las variables que aportan a la valoración social de la labor docente (Unesco, 2016; 2017).

Desde esta perspectiva, en Colombia la formación docente tomó cierta relevancia. Por ejemplo, y solo por mencionar una experiencia, desde 2012 el programa de transformación educativa Todos a Aprender (PTA), desarrolló acciones de acompañamiento in situ, por parte de sus pares, a los profesores de Educación Básica y más recientemente se piensa en ampliarlas a la Educación Media (Ministerio de Educación Nacional, 2018). Por otra parte, analizando la situación de la educación en el país, el polémico estudio Tras la excelencia docente: cómo mejorar la calidad de la educación para todos los colombianos (Fundación Compartir, 2014) insistió en la necesidad de promover la formación docente como tema fundamental de la política pública.

En el Plan Nacional de Desarrollo 2014-2018 se estableció: "la excelencia docente como línea estratégica para la política educativa" (Departamento Nacional de Planeación, 2014, p. 87), en el mismo plan se propuso brindar formación docente de alto nivel: "la formación de los docentes en doctorados y maestrías" (p. 97) a tal punto que en el 
documento se estableció que: "se ofrecerá apoyo a docentes para la formación avanzada en programas nacionales e internacionales" (p. 98). De esta forma, el que se enunciara en un plan de desarrollo la necesidad de formación docente puso en la agenda pública la necesidad de diseñar políticas claras al respecto. Los anteriores enunciados se pueden leer en clave de los requerimientos y retos que se vislumbran en el sistema educativo colombiano, donde se destaca como punto importante: "mejorar las prácticas docentes en todos los niveles. Las mayores expectativas para la profesión docente deberían acompañarse de oportunidades adecuadas de formación y remuneración" (OCDE, 2016, p. 5) con miras a mejorar la calidad de la educación, tema necesario para promover el crecimiento económico (OCDE, 2017).

De esta manera, articulado o no a los requerimientos internacionales, se generaron algunas iniciativas importantes de política pública en lo relacionado con la formación de profesores que desempeñan su labor en la educación básica y media, en el sector oficial. Una de ellas se originó en la Alcaldía de Bogotá, donde se propuso en el Plan Sectorial de Educación 2012-2018 el mejoramiento profesional de los docentes y directivos docentes, por medio de programas de formación de excelencia, buscando beneficiar a más de 9000 docentes con programas de postgrado de alto nivel, en alianza con algunas universidades de Bogotá. Esta formación posgradual debe conducir a transformaciones pedagógicas en el aula, la escuela y el territorio (Secretaría de Educación del Distrito de Bogotá, 2012). De igual manera, en 2016 en el plan de desarrollo de la Alcaldía de la ciudad 2016-2020 se prometió que "más de 11000 maestros participarán en los diferentes programas de formación desarrollados en el marco de la Red de Innovación al Maestro, incluyendo estudios post graduales a nivel nacional y pasantías a nivel internacional en universidades de alta calidad" (Alcaldía Mayor de Bogotá, 2016, p. 20). Así, docentes del sector oficial de Bogotá iniciaron y culminaron procesos de formación en especializaciones, maestrías y doctorados en educación en diferentes universidades capitalinas. Asimismo, diversas secretarías de educación del país fortalecieron la formación de sus maestros.

Por su parte, el Ministerio de Educación Nacional desarrolló estrategias formativas de alto nivel posgradual para profesores del sector oficial, denominadas becas para la excelencia. Estas consisten en un crédito beca, cien por ciento condonable, y con las que se buscó que "al cursar la maestría los docentes fortalecen sus competencias profesionales para enseñar contenidos específicos de sus disciplinas, y los procesos de reflexión-acción sobre problemas y situaciones del aula y la institución" (Ministerio de Educación Nacional, 2016); de esta manera, se ha apoyado la formación continuada de profesores, vinculándolos a diferentes programas de maestría del país. 
Con este contexto, varios profesores del sector oficial de la capital colombiana Ilegaron a cursar la Maestría en Educación de la Universidad de Santo Tomás de Aquino, en Bogotá. El equipo de profesores de la maestría que los trabajos de grado de estos maestros se desarrollarían con el horizonte metodológico de sistematización de experiencias, durante los años comprendidos entre 2012 y $2017 .{ }^{1}$ Ahora bien, en este trabajo nos centraremos en la experiencia compartida de una profesora y de un profesor (tutores) que tuvieron a cargo una cohorte que inició su proceso formativo en 2014 y lo culminó en 2016. Dicho grupo estaba conformado por 23 estudiantes, profesores todos en ejercicio adscritos a la Secretaría de Educación de Bogotá. Los tutores trabajaron como dupla de asesoría y formación en investigación de los estudiantes.

\section{Discusión conceptual}

La investigación social no se puede considerar como una simple acumulación de técnicas, sino, en sentido más amplio, como una práctica (Callejo, 2001). Con este contexto, la sistematización de experiencias, como ruta de investigación social, genera grandes tensiones en el campo de las ciencias sociales, especialmente porque se ponen en juego diversas prácticas sociales; por ello, esta naciente tradición permite desarrollar, como lo recuerdan Delgado y Gutiérrez (1999), la pluralidad de discursos y narrativas de diferentes actores sociales. Ahora bien, en la sistematización de experiencias se deben considerar dos conceptos relevantes: el de práctica y el de experiencia, constructos teóricos que abordaremos brevemente para aportar a la configuración teórica sobre la sistematización de experiencias.

El concepto de práctica suele estar emparentado con los asuntos técnicos del hacer; lo anterior ha Ilevado a que se privilegie una comprensión instrumental sobre dicha concepción, en diferentes esferas del saber y, especialmente, en el ámbito educativo:

[...] en una cultura que ha subordinado la excelencia de la práctica a la eficacia institucional, es de esperar que el debate educativo se reduce a un debate mundano técnico, en el que preguntas no-técnicas, sobre cómo lograr la excelencia en búsqueda de los bienes internos a la educación, ya no se hacen. (Carr, 2004, p. 68)

De esta manera, la noción de práctica queda anclada a la descripción técnica e instrumental sobre el cómo hacer algo y, a la vez, suele referirse a un conjunto de acciones que se oponen a lo teórico.

1 En este periodo se graduaron 122 estudiantes del convenio con la Secretaría de Educación de Bogotá. 
Sin embargo, en oposición a un enfoque instrumental de la práctica, como el que parece imperar, en el presente trabajo se ha asumido la práctica como:

[...] cualquier forma coherente y compleja de actividad humana cooperativa, establecida socialmente, mediante la cual se realizan los bienes inherentes a la misma mientras se intenta lograr los modelos de excelencia que le son apropiados a esa forma de actividad y la definen parcialmente, con el resultado de que la capacidad humana de lograr la excelencia y los conceptos humanos de los fines y bienes que conIleva se extienden sistemáticamente. (Maclntyre, 2009, p. 223)

Se trata, pues, de comprender la práctica como un sistema complejo de acciones e interacciones de los sujetos, orientado hacia uno o diversos fines, que comportan relaciones de coordinación y subordinación, regulado por normas de diferentes tipos y situado en un contexto que lo influye y le da significación y que él busca influenciar:

[...] sin sujeto no hay práctica y sin práctica no existe sujeto [...] el sujeto es propiamente sujeto de la acción y a menudo sujeto con otros, de esta forma la práctica permite al sujeto inscribirse en el mundo por iniciativa, en ella se devela y se transforma el sujeto y el mundo. (Nadeau, 2004, p. 216)

Podríamos decir, en consecuencia, que solo existe la práctica cuando hay practicante, como se explicita en prácticas y discursos (Martínez-Posada, Hernández-Molina N. y Hernández-Molina L. M., 2019).

De esta manera, a la práctica le es inherente el saber técnico, pero se relaciona más con una dimensión ética del actuar. Dicha situación se denomina como saber práctico — como el conjunto de acciones que se orientan por las decisiones éticas para actuar que posee un individuo. De esta forma, la phrónesis evoca un saber oportuno y eficaz del que dispone el ser humano y, en consecuencia, no remite primariamente a la teorización, sino que nace de las prácticas (Aubenque, 2009). Este saber lo identifica Aristóteles (1985) como un saber actuar, que es equivalente a la experiencia: ${ }^{2}$ la "phrónesis es el nombre de Aristóteles para la sabiduría y a la experiencia" (Flyvbjerg, 1991, p. 102). Desde esta perspectiva existe una delgada línea entre aquello que es la práctica y eso que es la experiencia, pues el practicante solo lo es mediante la experiencia y esta solo se cultiva por las prácticas del practicante.

2 Para profundizar en este planteamiento véanse: Aubenque (2009), Barragán (2015b), López (2018). 
Por su parte, el concepto de experiencia posee diversas entradas. A juicio de Martin Jay (2009), es uno de los conceptos filosóficos más difíciles de manejar, pues en una definición definitiva

[...] sería insensato decidir de antemano que ciertos significados son correctos y otros no [...] cuando una palabra ha tenido una historia tan larga y compleja como el término "experiencia", no es posible hacer justicia a sus avatares mediante un prematuro cierre semántico. (p. 25)

Sin embargo, al pensar aquello que puede llegar a significar la experiencia, esta adquiere un carácter dual pues se relaciona tanto con el mundo exterior como con la interioridad y devela la tensión epistemológica de buscar en ella un fundamento último del conocimiento. Pensar sobre la experiencia altera las rutas investigativas de las diferentes disciplinas, pero especialmente lanza los cuestionamientos sobre aquello que significa tener experiencia y a la manera como diferentes pensadores lo han resuelto ya sea por vía inductiva o deductiva (Barberousse, 1999; Eikeland, 2008, Perreau, 2010), al punto de entenderse la experiencia y la práctica como dimensiones coexistentes en el saber práctico que caracteriza al ser humano (Barragán, 2015b; Barragán, Martínez y Quiroga, 2018; Flyvbjerg, 2001).

Ahora bien, tener experiencia significa haber experimentado. Gadamer (1996; 2002) fijará la mirada en la historicidad humana e insistirá en que si alguien es experimentado en algo, no significa que conozca eso de manera definitiva, sino que por el contrario se abren las posibilidades para reconfigurar aquello que se experimenta: "una persona experimentada no es una persona dogmática. [La experiencia] nos permite abrirnos a cosas nuevas" (Gadamer, 2002, p. 52); ese individuo experimentado pone su mirada en sus experiencias de vida (Grondin, 2018; 2014). En esta medida, alguien experimentado es aquel que asume la experiencia como parte de su autocomprensión vital y actúa en consecuencia con eso asumido. En este sentido, la experiencia en sí misma no existe, sino que se convierte en un constructo dotado de significados, por aquellas subjetividades que se asumen como experimentadas en un conjunto de experiencias y, en consecuencia, pueden actuar en esos marcos de referencia en los que ellos se destacan como experimentados. Las subjetividades experimentadas pueden llegar a eventualizar (evento experimentado) una situación, en la que se visibilizan los sujetos: "eventualizar no es únicamente inventarse un acontecimiento, es mostrar cómo en un momento histórico un determinado enunciado se entendió en sus discursos y sus prácticas, cómo este cambio en sus límites históricos es establecer la transformación de los saberes" (Martínez, 2015, p. 10), es decir, se erige un acontecimiento epocal (Gadamer, 1998; 2001; 2007); un acontecimiento que marca época y que, por 
su particularidad, se puede recordar existencialmente. Aquello que marca época se puede eventualizar, así se convierte en un acontecimiento que puede ser narrado.

Con este preámbulo, es importante decir que la sistematización de experiencias, poco a poco, ha encontrado un fructífero enraizamiento en el trabajo con comunidades y, a la vez, emerge como ruta alternativa de investigación social participativa en la que se aborda la experiencia de una comunidad para aprender de lo acontecido, para fortalecer las posibilidades presentes $y$ futuras. Se trata de una

[...] interpretación crítica de una o varias experiencias, que, a partir de su ordenamiento y reconstrucción, descubre o explicita la lógica del proceso vivido, los factores que han intervenido en dicho proceso, cómo se han relacionado entre sí, y por qué lo han hecho de ese modo. (Jara, 1994, p. 70)

Este asunto, en todo caso, no se queda en la llana enunciación de lo que se ha eventualizado, sino que trasciende en pro de la transformación y la subjetivación política, en la que se llegan a reconfigurar imágenes del sujeto, pues este "está allí arrojado como construcción hegemónica y es vital reconfigurarlo desde las posibilidades del creer y el inventar. Como productos socioculturales creemos aquello dado, pero como posibilidad, los seres humanos estamos Ilamados a inventar" (Barragán y Sánchez, 2017, p. 76).

No sobra recordar que la sistematización de experiencias tiene sus raíces en la educación popular. Allí, los actores se convierten en el centro de la acción formativa, renunciando a cierta institucionalidad establecida y paralizada por la comprensión del ejercicio educativo en una sola vía. En la educación popular el saber proviene y vuelve a las comunidades en un claro ejercicio de reconocimiento del otro (Rodríguez, 1989; Sime, 1991; Torres, 2012; 2013); cabe decir que la sistematización de experiencias es originaria de América Latina (Verger i Planells, 2007). En este contexto, como horizonte investigativo, ${ }^{3}$ poco a poco ha conquistado el campo de indagación de las ciencias sociales, a tal punto que la apuesta política de este estilo de investigación se traduce en la posibilidad de generar teoría en la que diversos investigadores, especialmente latinoamericanos, han configurado un auténtico campo de saber (Carvajal, 2007; Cifuentes, 1999; Jara, 1994; 2001; 2012; 2014; Mejía, 2010; Torres, 1998). En este campo de indagación, se busca producir conocimiento a partir de la intervención

3 En ciertos círculos académicos se sostiene que la sistematización de experiencias no es propiamente un tipo de investigación, sino una manera de intervenir la realidad para recopilar datos sobre una situación dada. Para los efectos de este escrito, asumimos la sistematización de experiencias como una opción metodológica investigativa en la que los sujetos, con semánticas y metodologías propias, llegan a producir conocimiento. 
en la realidad, por medio del empoderamiento de las comunidades. Como se aprecia, diversos investigadores también presentan la sistematización de experiencias con diferentes matices, y existen tantas propuestas como autores que intentan delimitarla; las cuales, en general, no provienen propiamente de la pedagogía. Cifuentes (1999), a su vez, recuerda la clara intencionalidad política de la sistematización de experiencias que se realiza en la educación popular, mientras que en el trabajo social se asocia más a la producción de conocimiento académico. También, como asunto problemático, asoma el hecho de que mucho de lo que se llega a Ilamar sistematización de experiencias termina pareciéndose "más a informes de trabajos" (Barnechea y Morgan, 2010), desdibujándose la intencionalidad, el rigor y el alcance de esta postura investigativa.

Ahora bien, es claro que esta ruta metodológica no aparece, en primera instancia, en el campo canónico de la investigación educativa y/o pedagógica, pero se han realizado intentos de trasladar sus procederes a los espacios de formación escolar, especialmente en lo relacionado con las prácticas de profesores. De esta manera, la sistematización de experiencias es una oportunidad para configurar rutas de producción de conocimiento y, a la vez, transformar las realidades educativas.

Con este contexto, vale la pena recordar que Jara, quien es un referente importante en educación social y popular en el contexto latinoamericano, recuerda que "el ejercicio de sistematizar experiencias es un ejercicio claramente teórico; es un esfuerzo riguroso que formula categorías, clasifica y ordena elementos empíricos; hace análisis y síntesis, inducción y deducción; obtiene conclusiones y las formula como pautas para su verificación práctica" (Jara, 1997, p. 6). De esta manera, al trasladar lo propuesto por este autor al campo educativo se puede asumir que el proceso de sistematización se relaciona con experiencias relevantes — que pueden acontecer en espacios escolarizados o no escolarizados - de las cuales pueden emerger datos, que al ser organizados sistemáticamente, permiten encontrar regularidades que posibilitan procesos metodológicos. No sobra decir que lo relevante hace referencia al cumplimiento de los alcances pedagógicos y sociales propuestos, que sobre la experiencia vive y asume el actor educativo. Por ello, la sistematización no puede ser, en modo alguno, la simple organización sistemática de lo acontecido, pues esta no es solo:

» Narrar experiencias. Aunque esto sea útil para sistematizar, se debe ir mucho más allá de la narración.

» Describir procesos. Aunque es necesario hacerlo, se debe pasar del nivel descriptivo al interpretativo.

»Clasificar experiencias por categorías comunes. Puede ser una actividad que ayude al ordenamiento, pero no agota la necesidad de interpretar. 
» Ordenar y tabular la información sobre la experiencia. La sola tabulación no basta.

» Hacer una disertación teórica ejemplificando con algunas referencias prácticas porque no sería una conceptualización surgida de la interpretación de los procesos. (Jara, 1996).

También Alfredo Ghiso invita a ver el sistematizar como un "esfuerzo consciente de capturar los significados de la acción y sus efectos; como lecturas organizadas de las experiencias, como teorización y cuestionamiento contextualizado de la praxis social, para poder comunicar el conocimiento producido" (Ghiso, 1998, p. 12), por ello la distancia temporal y la misma experiencia permiten configurar una práctica relevante culminada o decantada, para considerarla en un marco simultáneo de sucesos y de esta forma reflexionar sobre ella. Este tema resulta atractivo para pensar sobre el quehacer de diferentes actores sociales:

[...] la sistematización como un tipo de reflexión sobre la práctica social o educativa que posee una intencionalidad, pertinente a un contexto particular y que no supone pensar en un sentido puramente abstracto, sino pensar acerca de algo: un quehacer, una experiencia social contextuada. Es un tipo reflexión crítica que se puede hacer sobre todos los componentes e interrelaciones de la experiencia; dando cuenta y comprendiendo las situaciones prácticas, los modos de definir, entender, valorar y resolver un problema social. (Ghiso, 2006, p. 82)

Por otra parte, Lola Cendales $(1996 ; 2000)$ plantea que la sistematización de experiencias permite generar inclusión, reconocimiento e interlocución entre los actores que intervienen en la sistematización. Lo anterior hace que se complejice la lectura de la realidad y se potencien las capacidades investigativas y pedagógicas, conceptuales y metodológicas de sujetos e instituciones involucradas en los procesos de sistematización. Para ella, "agenciar procesos de formación en los sectores populares demanda investigar con rigurosidad y sistematizar la riqueza enorme de prácticas que viajan en este país, a pesar de la adversidad y el escepticismo" (Ortega y Torres, 2011, p. 354). En esta visión el empoderamiento de participantes y constructores de la experiencia es fundamental para ir más allá de su simple reconstrucción histórica.

De manera especial, Alfonso Torres (1996) insiste en que la sistematización de experiencias se ha convertido en un legítimo campo intelectual en que se ha erigido con un horizonte de investigación cualitativa. Resalta el que esta opción de indagación debe llevar la participación de la comunidad y la transformación social, con miras a construir conocimiento a partir de un nosotros compartido, en el que el saber no es propiedad del intelectual. 
De forma análoga, María Mercedes Barnechea y María de la Luz Morgan (2010) reconocen que "La sistematización no se refiere a cualquier acción, sino a la que tiene lugar en el marco de proyectos y programas de desarrollo, es decir, de intervenciones intencionadas, con objetivos de transformación de la realidad" (p. 100), proyectos que necesariamente se anclan a la transformación social de manera organizada e intencionada. Para estas autoras, la experiencia es propiamente lo que pasó con el desarrollo mismo del proyecto y en cuyos ajustes los cambios "expresan descubrimientos que van emergiendo en la práctica: conocimientos nuevos sobre el contexto o una profundización de lo que ya se sabía sobre los problemas y las personas involucradas" (Barnechea y Morgan, 2010, p. 101).

De ahí que la sistematización de experiencias posee en sí misma lógicas propias que le permiten promover la transformación social de las comunidades, por medio el empoderamiento político a la vez que se produce conocimiento. De esta manera, se hace investigación rigurosa impactando la realidad:

La sistematización crea nuevos conocimientos a partir de la experiencia concreta, pero, en especial —en la medida en que su objeto de conocimiento son los procesos sociales y su dinámica- permite aportar a la teoría algo que le es propio: explicaciones sobre el cambio en los procesos. Con base en estas características propias, como ejercicio intelectual que permite elaborar conocimientos desde lo cotidiano y explicarse los factores de cambio en los procesos, es que la sistematización puede contribuir de forma decisiva a la recreación y a la construcción de teoría, dinamizando dialécticamente la relación entre el conocimiento teórico ya existente — como expresión de saber acumulado- y los nuevos conocimientos que surgen de las nuevas e inéditas situaciones de las experiencias que vivimos. Este es un aporte propio de la sistematización. (Jara, 1997, p. 6)

Hasta aquí hemos presentado aquello que diferentes autores proponen respecto a la sistematización de experiencias como una ruta importante para rescatar lo que sucede con una colectividad y, con base en ello, configurar transformación social y producir conocimiento. Intentando ir más allá, asumimos una caracterización propia sobre sistematización de experiencias - que en todo caso emerge también de la experiencia de los autores del artículo al orientar trabajos de grado con este enfoque-, al menos en los siguientes elementos:

» El objeto mismo de la sistematización es la experiencia; por ello, cobra importancia establecer qué se entiende por experiencia ya que de esa comprensión deriva el alcance de aquello que se sistematiza. Por ello, la pregunta ¿cuál es la experiencia? adquiere diversas dimensiones. 
» La experiencia es un asunto que viven las subjetividades experimentadas; son ellas las que dan cuenta de lo experimentado.

» Práctica y experiencia pueden connotar aspectos diferentes en el momento de sistematizar aquello acontecido. No obstante, pueden comportarse como conceptos sinónimos, de ahí que resulte importante establecer sus límites, alcances, encuentros y matices.

» La experiencia que se sistematiza puede tener diversos momentos, puede ser algo ya acontecido o bien puede tratarse de un fenómeno en marcha.

» Sistematizar implica poner en un orden lógico y coherente con algo acontecido. Sin embargo, no se puede quedar en la simple organización de los acontecimientos para poderlos categorizar, entender e investigar.

» La sistematización de experiencias tiene como finalidad principal el que los protagonistas de la experiencia puedan empoderarse de lo que se ha constituido como prácticas colectivas, para que, en la organización sistemática de lo acontecido, puedan reconfigurar su presente y proyectarse metas futuras.

»La experiencia colectiva es la fuente primordial de la sistematización; esto Ileva a generar empoderamiento en los actores.

» La transformación política es la finalidad última del ejercicio de sistematización.

» La sistematización de experiencias debe producir saberes.

» Como opción de investigación social, ha de ser sistemática y rigurosa.

Ahora bien, retomando aquello planteado al inicio de esta sección, vale la pena arriesgar una apuesta teórica. La sistematización de experiencias no se reduce a la mera anunciación de lo sucedido y, más puntualmente, le es imposible llegar a captar lo eventualizado. En la sistematización lo máximo que se alcanza es a tratar de captar la profundidad experiencial que logran narrar quienes han experimentado una determinada suerte de sucesos; en consecuencia, se puede llegar a reconocer las subjetividades experimentadas, que en el diálogo conjunto intentan transformar sus propias realidades. Este es, a lo sumo, el anhelo de esta opción metodológica: la transformación conjunta mediante la construcción de un nosotros que produce saber; es decir, se configura la comunidad de los experimentados, quienes no son experimentados sobre todo, sino sobre ciertas prácticas concretas que se mejoran con la experimentación misma. 


\section{Desarrollo metodológico}

La investigación a la que se ancla este trabajo es de corte cualitativo y tuvo por objetivo comprender las prácticas de formación en investigación de profesores-investigadores de la Maestría en Educación de la Universidad Santo Tomás, Colombia. De manera particular lo que se presenta en este artículo corresponde a una sección de esta investigación, donde dos docentes (tutores) realizaron un ejercicio comprensivo (Creswell, 1997; Geertz, 2009; Huberman y Miles, 1991) sobre sus propias prácticas al orientar trabajos de grado de nivel de maestría entre 2014 y 2016. Dichos trabajos se desarrollaron con el enfoque de sistematización de experiencias como ruta metodológica; el ejercicio reflexivo de los tutores derivó en una propuesta de acompañamiento de trabajos de grado siguiendo el mencionado enfoque metodológico. A partir del proceso de acompañamiento, los tutores realizaron registros reflexivos sobre sus prácticas de tutoría de los seis trabajos de grado acompañados (tabla 1); estos registros eran discutidos con otros profesores-investigadores de la maestría. Tales acciones permitieron desarrollar procesos de reflexión y acción sobre la práctica de asesoría de los tutores desde una perspectiva de reflexibilidad crítica (Carr, 2004) y centrando la mirada en la comprensión del saber práctico (Barragán, 2015b), desde donde se entendió el concepto de práctica.

\section{Tabla 1.}

Relación de trabajos de grado

\section{Título}

Número de participantes

Sistematizar y transformar las propias prácticas educativas a través de la comprensión y reflexión del concepto de formación integral

4

Sistematización de experiencias con miras a generar un saber pedagógico a través de una comunidad de práctica que reflexiona sobre la comunicación humanizante, la persona y las prácticas del maestro en la escuela

La transformación de nuestras prácticas educativas en relación con las emociones y el proceso de enseñanza-aprendizaje

Sistematización de la experiencia "La lúdica en el aula de clase por medio del juego del ajedrez"

Sistematización de la experiencia "'Colombianito', una metodología para promover desarrollos y aprendizajes en edad preescolar"

Sistematización de la experiencia "La huerta escolar como ambiente de aprendizaje".

Total

Fuente: elaboración propia
5

5 3 3 3 23 
Adicionalmente, con los 23 estudiantes se desarrollaron cuatro grupos de discusión. Como lo plantean Canales (1999), Callejo (2001) y Gutiérrez (2008), el grupo de discusión promueve el que emerjan narrativas en las que aparecen las subjetividades que allí participan. En los grupos de discusión se presentaron varias preguntas generadoras que indagaban por la experiencia de los participantes al realizar sus trabajos de grado con el enfoque de sistematización de experiencias. De los grupos de discusión se escogieron cuatro participantes (uno por grupo) para realizar entrevistas a profundidad, en las que se indagó por otros aspectos que no hubiesen aparecido en los señalados espacios de discusión. A todos los participantes se les solicitó el consentimiento informado.

La información recolectada se abordó desde algunos elementos de análisis de contenido (Creswell, 1997; Huberman y Miles, 1991; Krippendorf, 1990; Navarro y Díaz, 1999), técnica que lleva a cuestionar "la estructura interna de la comunicación (composición, organización, dinámica) y el contexto en el cual se produce la información" (Galeano, 2009, p. 123). De esta manera, los tutores pudieron reconstruir sus ejercicios tutoriales y a partir de allí establecieron la estrategia utilizada al orientar trabajos de grado de nivel de maestría, la cual denominaron Itinerario formativo e investigativo en sistematización de experiencias (Ifise). A continuación se explican las fases de esta estrategia.

\section{Alistamiento: presentar la ruta}

Lo primero que se hizo fue presentar a los estudiantes la propuesta de investigación en sistematización de experiencias que, como suele suceder, generó inicialmente algunas tensiones debido al poco conocimiento que se tenía sobre esta opción metodológica; esto generó una especie de desconfianza en aquellos maestrantes que comprendían y conocían las características de la investigación cuantitativa o cualitativa más tradicional. En consecuencia, fue necesario ampliar las concepciones sobre los tipos de investigación cualitativa, las variedades metodológicas, ya que generalmente los espacios introductorios de metodología solo presentan las diferencias entre lo cualitativo y lo cuantitativo, pero no se profundiza sobre sistematización de experiencias. Como parte del alistamiento, desde la primera sesión, a todos los participantes se les pidió ubicar una experiencia que resultara significativa para ser sistematizada. De igual manera, también se les pidió realizar un diario de campo sobre sus propias prácticas de aula, para encontrar una experiencia compartida que pudiese ser sistematizada y que se construyera durante el proceso. 


\section{Encontrar regularidades: pensar la investigación}

Establecidos los aspectos iniciales de conceptualización, sobre práctica y sobre experiencia, se consolidaron los equipos de investigación de estudiantes, partiendo de la premisa de que el aprendizaje se construye en colectivo y que el proceso de investigación no debe ser solitario sino comunitario. Los equipos de trabajo de estudiantes no se integraron por áreas de conocimiento, ni por niveles académicos, sino inductivamente a partir de sus intereses, para permitir acercarse a los aspectos más relevantes de sus prácticas; la conformación de los grupos se realizó por medio de un taller de Cartografía Social Pedagógica (CSP), la cual se puede entender como:

Una estrategia de investigación y acompañamiento en la que, por medio de la acción colectiva, se lleva a los participantes a reflexionar sobre sus prácticas y comprensiones de una problemática común, mediante el levantamiento de un mapa (cartografía) en el que se evidencian las problemáticas que acontecen en un territorio; en este caso, los territorios relacionados con las tensiones de las prácticas de enseñanza y de aprendizaje [...] Importa en esta estrategia el que los diferentes actores lleguen a acuerdos sobre las interpretaciones que emergen de las problemáticas socioculturales que se evidencian en el territorio. (Barragán, 2016, p. 256)

En este caso, el territorio se comprendió en relación con las realidades educativas en instituciones públicas en las que desarrollan las prácticas los estudiantes de la maestría. El proceso cartográfico permitió que estos - a pesar de estar ubicados en diversas áreas de conocimiento en su desempeño profesional, en diferentes niveles educativos e incluso en diversos lugares de la ciudad - identificaran puntos de encuentro a partir de sus prácticas, para ubicar las regularidades de su quehacer y, en consecuencia, establecer los puntos por intervenir para transformar sus prácticas y reconfigurar sus experiencias. A partir de este taller cartográfico se formularon las preguntas provisionales de investigación que luego darían forma a los objetivos de las investigaciones. Cabe mencionar que en esta propuesta las preguntas de investigación se enuncian de manera provisional, y solo se llega al diseño metodológico después de haber superado las etapas de aprender con los otros, visitar la teoría y visitar los contextos. Es entonces cuando tales peguntas se enuncian de manera definitiva para establecer los objetivos de investigación.

\section{Aprender con los otros: configurar comunidades de práctica}

Posteriormente, se hizo necesario realizar la contextualización de las prácticas sobre la cuales se planteaban las experiencias de sistematización por cada grupo de trabajo. Este ejercicio llevó a que los maestrantes generaran 
comunidades de práctica, las cuales favorecen que se aprenda entre pares y se reconfiguren las acciones concretas de transformación (Barragán, 2015a). Para facilitar la comunicación entre los diferentes grupos, las comunidades de práctica se desplazaron al ciberespacio en forma de un blog, donde se alojaron videos de los contextos escolares de las experiencias de los estudiantes y se podía discutir sobre lo que allí se presentaba.

Estas comunidades de práctica permitieron revisar constantemente lo que los diferentes grupos realizaban durante su proceso investigativo y posibilitaron centrar la mirada en las experiencias por sistematizar. En estos espacios se debatía acerca de las experiencias y los aspectos metodológicos de cada una de las investigaciones: "la sistematización de experiencias educativas, es claro que nos permite apropiarnos de nuestras prácticas, porque se investiga sobre las mismas y se complementa la teoría con la práctica. Esto se hace mejor cuando se dialoga con lo pares, quienes son nuestros mejores evaluadores y animadores" (estudiante 22).

\section{Visitar la teoría: aprender de lo escrito}

En paralelo con lo anterior, en los grupos de trabajo se avanzó en la elaboración del estado de la cuestión, mediante rastreos académicos en diversas bases de datos, buscando investigaciones sobre sistematización de experiencias educativas, con énfasis en los puntos de referencia sobre las comprensiones realizadas en estos trabajos y en las apuestas metodológicas desarrolladas en ellos. Estos procesos generaron aún más interés y reforzaron las concepciones sobre sistematización de experiencias abordadas en el proceso formativo.

Una vez realizado el estado de la cuestión, se profundizó en la fundamentación conceptual sobre sistematización de experiencias, apoyándose en los diferentes autores e investigaciones acerca de la temática y desde los aspectos metodológicos que pudieron encontrarse en el levantamiento del estado de la cuestión. Una nota característica de este proceso fue trabajar sobre experiencias en curso o creadas para este ejercicio investigativo - este último aspecto se convirtió en el elemento diferenciador- para que los estudiantes pudiesen establecer las experiencias por sistematizar; lo anterior obedeció a que "emergió una inquietud importante con la que nos encontramos como profesores que orientábamos la investigación: se trataba de revisar la concepción sobre experiencia que podía ser susceptible de ser sistematizada a partir de la historicidad de los maestrantes" (tutor 1).

La anterior decisión corresponde a entender que, en la sistematización de experiencias, estas, en algunos casos, pueden corresponder a acciones colectivas que, desarrolladas desde horizontes comunes, permanecen en el tiempo y en la memoria de una comunidad. De ahí el carácter y la 
potencia de empoderamiento social de los actores que participan de esta ruta metodológica, en la que se trasciende la recuperación de memoria al poner la atención en un sistema conformado por las acciones de un grupo de personas o de un individuo. Desde esta perspectiva,

[...] las experiencias pedagógicas de los maestrantes, en sentido estricto, no correspondían a las búsquedas y alcances de la metodología de sistematización de experiencias, pues las experiencias de estos maestros en ejercicio se centraban más en las acciones desarrolladas en su práctica y el sentido comunitario, en el mejor de los casos, emergía como una prolongación de la misma. (Tutor 2).

Estos aspectos permitieron generar la necesidad de plantear una postura conceptual sobre aquello que se podía entender por la práctica del docente, práctica pedagógica o práctica educativa y, repensar la relación entre práctica y experiencia. Entonces, como aspecto particular,

[...] encontramos que era necesario discutir y enfatizar sobre las tensiones que emergen al pensar aquello que pueda ser una experiencia pedagógica, en relación con las prácticas, pues el interés fundamental de todos los participantes en el proceso era el de la transformación y mejora de sus prácticas. (Tutor 2).

\section{Visitar los contextos: aprender de las experiencias}

En sincronía con la revisión conceptual desarrollada en el proceso investigativo de los estudiantes, se desarrollaron varias visitas en las que tutores y estudiantes se relacionaron directamente con el contexto y con los actores de las experiencias, no desde el punto de vista de un objeto de estudio, sino en la perspectiva de interrelación con la experiencia: "este acompañamiento que se realizó semestre a semestre permitió que todos aquellos que participábamos de manera directa o indirecta de la sistematización comprendiéramos aspectos de la misma que superaron las pretensiones investigativas" (tutor 1). Estas visitas permitieron comprender lo que los estudiantes estaban sistematizando y facilitaron el que los tutores reconfiguraran los aspectos metodológicos y teóricos según las necesidades de cada grupo. Esto posibilitó la subjetivación como investigadores, tal como lo afirma un estudiante:

[...] la sistematización de experiencias educativas significó para mí, el empoderamiento como investigador educativo y como transformador, modesto, pero trascendente de ambientes de aprendizaje, en torno al mejoramiento continuo del quehacer educativo, en donde el enfoque humanista determina el norte y el sentido último del proceso de enseñanza aprendizaje. (Estudiante 7). 


\section{Visitar la metodología: aprender a organizar}

Como se puede apreciar, la ruta de entrada no fue la teorización ni el diseño metodológico, como suele ocurrir en la investigación tradicional, sino que los diferentes grupos ajustaron sus referentes teóricos, en la medida en que revisaban sus prácticas y experiencias con sus propios instrumentos y con la mirada de otros colegas:

[...] no es fácil estar revisando autores y viendo la experiencia de uno y teniendo lo metodológico como algo en blanco y negro, me refiero a los objetivos, claro. Los compañeros de otros grupos nos dicen que estamos quedados, pero nosotros vemos que lo metodológico solo es una manera de organizar nuestra experiencia, eso es algo que quería decir. (Estudiante 4).

Como se ha mencionado, solo después de superar las fases anteriores, se ajustan de manera definitiva la pregunta de investigación — que ha estado presente de manera provisional desde el inicio-, los objetivos y las estrategias de recolección de información. De esta manera, el proceso no inicia con el diseño metodológico pues se recurre a ubicar las regularidades de las prácticas y experiencias comunes de los participantes y de allí emerge la experiencia compartida que se va a investigar.

En cuanto al proceso metodológico se realizaron varios ajustes ya que no solo era importante que los estudiantes generaran espacios de apropiación de técnicas e instrumentos de recolección y análisis de la información, sino que también se empoderaran de lo que significaba la sistematización de experiencias: "cada proceso investigativo de sistematización de experiencias, no obedece a una rigurosidad metodológica establecida [tradicional]; al contrario, cada sistematización de experiencias genera su propia ruta metodológica" (estudiante 10). De esta forma, el proceso reflexivo en lo relacionado con las prácticas, su desarrollo, el impacto en las comunidades, entre otros tantos elementos, permitió que los estudiantes no se asumieran como observadores externos del proceso, sino que se reconocieran como actores de este.

Ahora bien, como se trataba de un espacio de investigación en el contexto de una maestría en educación, poco a poco los maestrantes fueron adquiriendo habilidades propias de la investigación que pudiesen asumir en sus procesos de sistematización, para elaborar rigurosamente el informe final de investigación.

\section{Ajustar lo formal: buscar el rigor}

Con los anteriores elementos, se procedió a elaborar los informes de investigación con los que cada estudiante optaría al título de magíster en Educación. Como se mencionó, se escogió la ruta de la sistematización de experiencias: 
[...] desde [un] punto de vista metodológico es apropiado tener claro que sistematizar experiencias, no significa organización de datos, o experiencias, ni seguir una ruta metodológica específica, la sistematización de experiencias la concibo como un proceso investigativo, donde se tiene claro que el objeto de investigación son nuestras prácticas educativas, mediante un proceso de reflexión continuo. (Estudiante 2).

En consecuencia, al realizar y revisar el informe de investigación los estudiantes pudieron evaluar rigurosamente los aspectos vivenciales, teóricos y metodológicos que quedaron consignados en el texto escrito, lo cual fortaleció la calidad académica de sus trabajos. El rigor al investigar Ilevó a que los diferentes actores reconocieran su subjetivación como investigadores, tal como lo menciona una profesora: "Sí considero que la investigación tiene muchos pasos y nosotras solo hemos dado un pasito, sí somos investigadoras pero es necesario... necesitamos seguir, nosotras nos formamos con cada clase, en el día a día" (estudiante 23), y sigue otro maestro "al final de la maestría siento que he crecido como persona, pues ahora soy un mejor docente, pero sobre todo que ya no me da miedo investigar" (estudiante 12) o como comenta otro profesor: "creo que ya puedo seguir investigando desde mi propio salón de clases y especialmente hacerlo desde la sistematización de experiencias" (estudiante 15) y como ratifica una maestra: "también, yo quería decir que ellos [los tutores] nos ayudaron a asumir el papel de investigador, para así ayudar a otros a investigar [...] eso demandaría pues que uno investigue con mayor frecuencia, que uno lea, porque ya va a depender es de uno" (estudiante 11).

\section{Socializar: el deber ético}

Como parte del proceso se pidió a los maestrantes dar a conocer los resultados de la sistematización a las diferentes comunidades educativas donde desarrollaron los procesos investigativos. Estas acciones permitieron empoderar de una mejor manera a todos los miembros de las comunidades y, en especial, fortalecer las propias experiencias de los maestrantes: "actualmente he podido iniciar una propuesta pedagógica, en donde la investigación adelantada dentro del programa de la maestría en torno a la sistematización, ha dado sustento teórico y metodológico a la misma" (estudiante 21). Estas acciones de socialización se incluyeron en el documento final que se entregó para optar al título de magíster.

\section{Generar teoría: más allá de la sistematización}

Como asunto particular, se insistió en que los diferentes trabajos plantearan, a partir de lo sistematizado, nuevas rutas para comprender la educación y que no se quedaran en la descripción de lo acontecido. 
Un ejemplo de ello se expresa en el siguiente testimonio: "no existe experiencia sin práctica ni práctica sin experiencia, como tampoco no [sic] existe sistematización de experiencias sin reflexión sobre nuestras prácticas educativas" (estudiante 15). Los tutores buscaron que durante el proceso los maestrantes se subjetivaran como investigadores $y$, en consecuencia, arriesgaran posturas teóricas propias que nacieran de todo el proceso de formación en la maestría. Algunos grupos se atrevieron a proponer tímidas apuestas conceptuales sobre los problemas que los convocaban, por ejemplo, cuando una maestra afirmó: "ahora, ya me arriesgo a hablar por mí misma y no recurrir tanto a los autores, es decir, los autores son importantes (manejo algunos teóricos) pero nuestro grupo se arriesgó a proponer definiciones nuestras sobre pedagogía, didáctica o educación. Para eso nos sirvió nuestra maestría, para arriesgarnos a teorizar" (estudiante 9). De igual manera, para los tutores resultó un reto configurar, ellos mismos, teoría educativa y, en este caso, proponer una ruta en forma de itinerario.

Las anteriores fases fueron reconstruidas por los tutores a partir de su ejercicio reflexivo sobre la orientación de los trabajos de grado de los estudiantes de la maestría, aquello que emergió en los grupos focales y en las entrevistas. Al revisar sus prácticas como tutores encontraron una experiencia compartida de orientación académica:

[...] revisar lo que hicimos en estos años, ha permitido que podamos reconstruir algunas cosas que habían quedado en el olvido; fue un buen trabajo, creo. Además, nos motivó a proponer una estructura, un paso a paso del que fuimos conscientes, de manera detallada, cuando pudimos ver hacia atrás. (Tutor 2).

De esta forma, los tutores propusieron la estrategia Itinerario formativo e investigativo en sistematización de experiencias (Ifise); nombrar la estrategia de esta manera implica que, como itinerario, es un camino ya recorrido que puede ser andado por otros. Asimismo, como estrategia formativa e investigativa, aportar a la subjetivación como investigadores de quienes participan en ella. Finalmente, por las particularidades de la sistematización de experiencias, la estrategia posibilita una ruta particular de acercamiento a esta forma de indagación. Así, la ruta propuesta en la estrategia Ifise establece un paso a paso del proceso formativo y puede servir de guía en el momento de orientar trabajos de sistematización de experiencias en el campo de la educación. En la figura 1 se muestra una infografía que sintetiza los pasos descritos. 


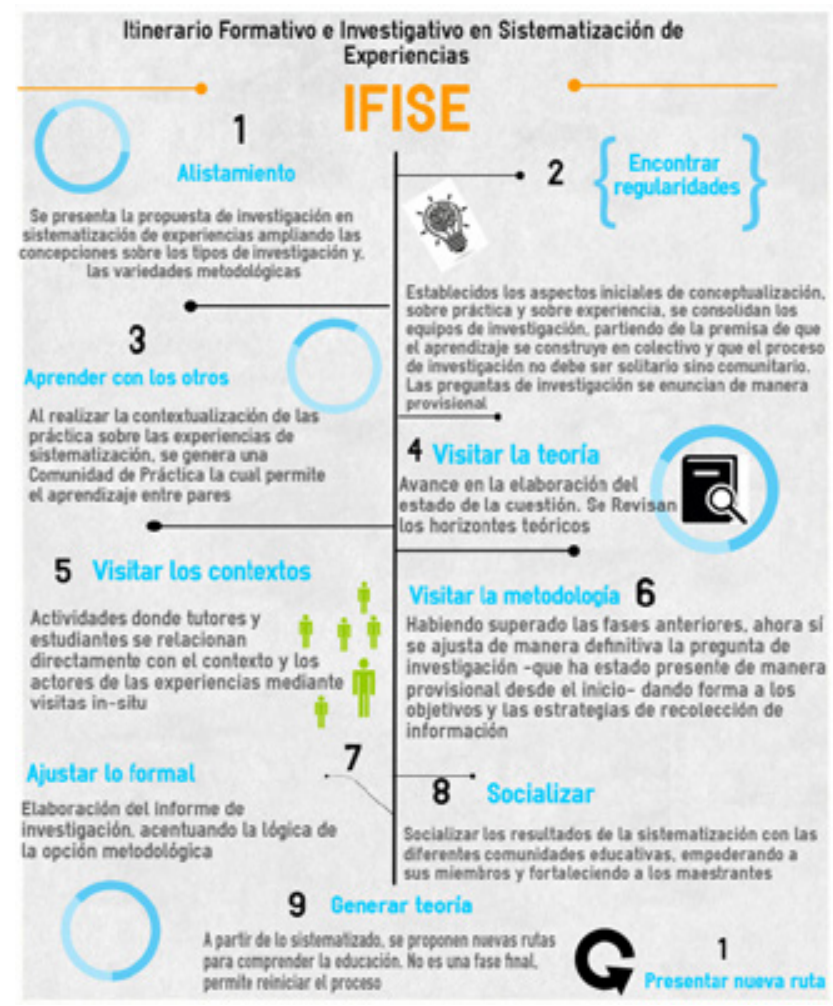

Figura 1. Infografia: Estrategia Ifise

Fuente: elaboración propia

\section{Resultados}

En el proceso de formación se incentivó, en los estudiantes, la apropiación del rol de investigadores, mediante la ruta metodológica planteada por los tutores, quienes hicieron especial énfasis en formar para la toma de decisiones en los procesos investigativos. Se ubicó al estudiante como centro de las dinámicas de la investigación.

Se aportó a la configuración de la metodología de sistematización de experiencias como una opción de investigación en educación. De esta manera, la transición de la sistematización de experiencias a los procesos de educación posgradual implicó incluir, modificar y ampliar rutas de comprensión de la metodología y de los contextos a los cuales tradicionalmente se le asocia. Como resultado de la experiencia recobrada de los tutores al orientar los trabajos de grado, se consolidó una estrategia de trabajo y promoción de la sistematización de experiencias en el campo de la educación superior. 
La apropiación de los elementos metodológicos de la sistematización de experiencias por parte de los maestrantes tuvo varios nodos de comprensión del proceso.

El primero de ellos fue la dificultad de involucrarse en una ruta investigativa poco conocida para ellos, como lo era la sistematización de experiencias. Esto debido a la poca experiencia investigativa de los estudiantes y a que quienes la tenían la habían adquirido en un enfoque cuantitativo o con algunas idas genéricas de investigación cualitativa.

El segundo nodo identificado se relaciona con la dificultad de ubicar la experiencia misma, no solo en términos de las delimitaciones necesarias para el proceso de sistematización sino en la conceptualización frente a la divergencia entre experiencia y práctica docente. Este asunto es vital para sistematizar aquello que los estudiantes pudiesen entender crítica y rigurosamente como experiencia.

El tercer nodo que se vislumbró tuvo que ver con superar la descripción de las situaciones que enmarcan la experiencia para darle sentido en términos de producción de conocimiento y plantear los elementos relevantes de esta, más allá de la narración anecdótica. Al estar en proyectos de investigación, las diferentes experiencias sistematizadas ganaron en rigor académico $y$, sobre todo, en tales proyectos se puede dar cuenta de los procesos seguidos en la sistematización y, desde luego, en los resultados alcanzados.

Otro aspecto fundamental fue el que la experticia de los tutores, sumada a las vivencias de los maestrantes, permitió consolidar comunidades de práctica y así superar colectivamente la incertidumbre y los azares del proceso metodológico.

En consonancia con lo anterior, los tutores diversificaron el proceso metodológico de acompañamiento y ampliaron las propias comprensiones sobre sistematización de experiencias. Esto permitió crear la estrategia que denominaron itinerario formativo e investigativo en sistematización de experiencias (Ifise). La principal característica de esta estrategia es que no se inicia, en sentido estricto, con el diseño metodológico, como usualmente se hace en la investigación tradicional. En consecuencia, las preguntas de investigación son provisionales y solo alcanzan su forma definitiva, dando origen a los objetivos, cuando se ha superado las siguientes fases: pensar la ruta, encontrar regularidades, aprender con los otros, visitar la teoría, visitar los contextos. Quienes lo consideren pertinente pueden asumir esta estrategia como una ruta replicable para orientar este tipo de trabajos.

La estrategia Ifise permite que tutores y estudiantes puedan abordar los procesos investigativos desde las prácticas, visitar la teoría y volver a las prácticas en un decurso continuo. Esto posibilita un compromiso mayor con la formación en investigación, pues desde el principio aparece una 
conexión entre el ejercicio investigativo y las necesidades del contexto, rompiendo con los ejercicios tradicionales de abstraer una problemática a partir de la consulta teórica para ser revisada o corroborada en los contextos concretos. En términos generales, el estudiante no abandona lo que conoce (sus prácticas, experiencias y vivencias) para desarrollar su proceso investigativo; en consecuencia, se genera en ellos posibilidades de apropiación, interés constante y aumento de la experticia: se convierten en experimentados en investigación. De esta forma, los investigadores pueden llegar a sugerir rutas de transformación de los contextos y a la vez, proponer conocimiento desde la sistematización de experiencias.

La estrategia Ifise, aun cuando se desarrolló con estudiantes de maestría, puede utilizarse en diferentes niveles formativos.

Todos los participantes en el proceso reconocieron que la sistematización de experiencias les permitió configurarse como investigadores y, sobre todo, transformar sus prácticas. De este modo se le dio alcance a lo solicitado por los entes gubernamentales, quienes requerían que los procesos formativos de estos profesores llevaran a recontextualizar sus propias prácticas para mejorar su docencia y aportar a la transformación del contexto.

\section{Conclusiones}

Las características de la sistematización de experiencias en el ámbito de la educación popular y del trabajo social son relevantes y potentes para ser utilizadas en la investigación educativa y pedagógica; sin embargo, se deben generar nuevos marcos de referencia para estos campos de saber, especialmente porque la indagación sobre las experiencias educativas y/o pedagógicas quedan constreñidas al discurso anecdótico de las prácticas del profesor y se olvidan las dimensiones colectivas en las que nace. De ahí que también una experiencia deba constituirse para así sistematizarse y no solo se recurra a la sistematización de experiencias en curso.

La forma como se ha agenciado e investigado una comprensión sobre la sistematización de experiencias en el campo educativo formal parece ser insuficiente pues los conceptos de experiencia y práctica se dan, la mayoría de las veces, por sentados y definidos unívocamente, por lo que se hace necesario abordarlos y estudiarlos rigurosamente cada vez que se inicien este tipo de trabajos.

Las comprensiones epistemológicas y ontológicas sobre la sistematización de experiencias necesitan de más marcos de referencia, pues los actuales resultan insuficientes. 
Para aportar a la configuración teórica de la sistematización de experiencias se debe considerar que la experiencia es importante, pero lo más valioso es cómo los seres humanos llegan a subjetivarse en el proceso de sistematización. En consecuencia, la experiencia produce personas experimentadas en algo, es el eje mismo de la comprensión de la práctica y la propia experiencia. De este modo, el discurso de las subjetividades experimentadas es enunciado en la experiencia, convirtiéndola en una eventualización, es decir, la experiencia misma es un acontecimiento epocal, que al estar situado en un territorio emerge como espacios de subjetivación y transformación.

Un tutor experimentado es aquel que ha tenido la experiencia tutorial, no desde el libro sino desde las prácticas; en consecuencia, se requiere de ejercicio de sistematización de la experiencia de los tutores de investigación, para producir teoría y generar aprendizajes. Un ejemplo de la anterior es la estrategia Itinerario formativo e investigativo en sistematización de experiencias (Ifise), la cual es una primera apuesta y debe seguirse mejorando.

Se hace indispensable y urgente, desde la formación de maestría, contribuir a la transformación de las tradiciones investigativas predominantes para diversificar las maneras de indagar en educación. Una ruta es la sistematización de experiencias, donde además de formarse en investigación se llega a modificar las prácticas.

\section{Referencias}

Alcaldía Mayor de Bogotá. (2016). Plan de desarrollo 2016-2020. Bogotá Mejor Para Todos. Bases del plan sector educación. https://www.educacionbogota.edu.co/portal_institucional/sites/default/files/inline-files/Plan_sectorial_2016-2020-.pdf

Aristóteles. (1985). Ética nicomáquea. Ética Eudemia. J. Pallí Bonet (Trad). Madrid: Gredos.

Aubenque, P. (2009). La prudence chez Aristote. París: Quadrige/PuF.

Barberousse, A. (1999). L'expérience. París: Flammarion.

Barnechea, M. M. y Morgan, M. D. (2010). La sistematización de experiencias: producción de conocimientos desde y para la práctica. Tendencias y Retos, 15, 97-107.

Barragán, D. (2015a). Las comunidades de práctica (CP): hacia una reconfiguración hermenéutica. Franciscanum, 57(163), 139-165.

Barragán, D. (2015b). El saber práctico, Phrónesis. Hermenéutica del quehacer del profesor. Bogotá: Universidad de La Salle.

Barragán, D. (2016). Cartografía social pedagógica: entre teoría y metodología. Revista Colombiana de Educación, 70, 247-285. 
Barragán, D., Martínez, J. y Quiroga, L. (2018). El campo hermenéutico para el desarrollo (CHD): Sobre el vulnerable como un "otro". Revista Internacional de Cooperación y Desarrollo, 5(2), 196-210. https:// halshs.archives-ouvertes.fr/halshs-02358286/document

Barragán, D. y Sánchez, N. (2017). Constitución de subjetividad: tensiones y emergencias. En J. Martínez (ed.), Dispositivos de producción de subjetividades: perspectivas conceptuales (pp. 61-76). Bogotá: Universidad de La Salle.

Callejo, J. (2001). El grupo de discusión: introducción a una práctica de investigación. Barcelona: Ariel.

Canales, M. Y. (1999). Grupos de discusión. En J. M. Delgado y J. Gutiérrez, Métodos y técnicas cualitativas de investigación en ciencias sociales. Madrid: Síntesis.

Carr, W. (2004). Philosophy and Education. Journal of Philosophy of Education, 38(1), 56-73.

Carvajal, A. (2007). Teoría y práctica de la sistematización de experiencias. Santiago de Cali: Universidad del Valle.

Cendales, L. (1996). Experiencias de sistematización. Revista Aportes. Sistematización de Experiencias. Búsquedas Recientes, 44, 98-121.

Cendales, L. (2000). El diálogo. Recorrido y consideraciones a partir de una experiencia. Aportes, 53, 45-63.

Cifuentes, R. M. (1999). La sistematización de la práctica del trabajo social. Buenos Aires: Lumen/Humanitas.

Creswell, J. (1997). Qualitative inquiry and research design: choosing among five traditions. California: Sage.

De Amicis, E. (2012). Corazón. Diario de un niño. México: Porrúa.

Delgado, J. M. y Gutiérrez, J. (1999). Métodos y técnicas cualitativas de investigación en ciencias sociales. Madrid: Síntesis.

Departamento Nacional de Planeación. (2014). Plan Nacional de Desarrollo 2014-2018. Todos por un Nuevo País. Tomo 1. Bogotá: Departamento Nacional de Planeación.

Eikeland, O. (2008). Aristotelian Phrónêsis, Aristotelian Philosophy of Dialogue, and Action Research. Bern: Peter Lang.

Flyvbjerg, B. (1991). Sustaining non-rationalized practices: Body-mind, power and situational ethics. An interview with Hubert and Stuart Dreyfus. Praxis International, 11(1), 100-120.

Flyvbjerg, B. (2001). Making social science matter: Why social inquiry fails and how it can succeed again. Londres: Cambridge University Press.

Fundación Compartir. (2014). Tras la excelencia docente: cómo mejorar la calidad de la educación para todos los colombianos. Bogotá: Fundación Compartir. 
Gadamer, H.-G. (1996). Entrevista con Hans-Georg Gadamer. Salud Mental y Cultura, s.d., 109-122.

Gadamer, H.-G. (2001). Verdad y método I . A. Agud y R. De Agapito (Trads.). Salamanca: Sígueme.

Gadamer, H.-G. (1998). Verdad y método ॥ . M. Olasagasti (Trad.). Salamanca: Sígueme.

Gadamer, H.-G. (2002). Gadamer in conversation; reflections and commentary. R. Palmer (Trad.). New Haven: Yale.

Gadamer, H.-G. (2007). El problema de la conciencia histórica. A Moratalla (Trad.). Madrid: Tecnos.

Galeano, M. E. (2009). Estrategias de investigación social cualitativa. El giro de la mirada. Medellín: La Carreta.

Geertz, C. (2009). La interpretación de las culturas. Madrid: Gedisa.

Ghiso, A. (1998). De la práctica singular al diálogo con lo plural. Aproximaciones a otros tránsitos y sentidos de la sistematización en épocas de globalización. Ponencia presentada en el Seminario Internacional sobre Sistematización de Prácticas de Animación Socio-Cutural y Participación Ciudadana. 12-14 de agosto, Medellín.

Ghiso, A. (2006). Prácticas generadoras de saber. Reflexiones freirianas en torno a la práctica de la sistematización. Educación y Ciudad, 11, $72-88$

Grondin, J. (2014). A la escucha del sentido. Conversaciones con Marc-Antoine Vallée. Barcelona: Herder.

Grondin, J. (2018). Del sentido de las cosas. La idea de metafísica. Barcelona: Herder.

Gutiérrez, J. (2008). Dinámica del grupo de discusión. Cuadernos Metodológicos 41. Madrid: Centro de Investigaciones Sociológicas.

Huberman, M. y Miles, M. (1991). Analyse des donnés qualitatives. Bruxelles: De Boeeck.

Jara, O. (1994). Para sistematizar experiencias. Una propuesta teórica y práctica. Lima: Tarea.

Jara, O. (1996). Tres posibilidades de sitematización: comprensión, aprendizaje y teorización. Aportes, 44, 7-22.

Jara, O. (1997). El aporte de la sistematización a la renovación teórico-práctica de los movimientos sociales. Medellín: Programa Coordinado de Educación Popular.

Jara, O. (2001). Dilemas y desafios de la sistematizaciòn de experiencias. Cochabamba: Seminario Asocam.

Jara, O. (2012). Sistematización de experiencias, investigación y evaluación: aproximaciones desde tres ángulos. Revista Internacional sobre Investigación en Educación Global y para el Desarrollo, 1, 56-70. 
Jara, O. (2014). La sistematización de experiencias: práctica y teoría para otros mundos posibles. Lima: Programa Democracia y Transformación Global. Consejo de Educación de Adultos de América Latina. Centro de Estudios y Publicaciones Alforja.

Jay, M. (2009). Cantos de experiencia. Variaciones modernas sobre un tema universal (trad. G. Ventureira) Buenos Aires: Paidós.

Krippendorf, K. (1990). Metodología de análisis de contenido. Teoría y práctica. Barcelona: Paidós.

Lledó, E. (2018). Sobre la educación. La necesidad de la literatura y la vigencia de la filosofía. Madrid: Taurus.

López, M. D. (2018). La hermenéutica filosófica de H.-G. Gadamer en búsqueda de la verdad. Madrid: Dykinson.

Maclntyre, A. (2009). Tras la virtud. Barcelona: Crítica.

Martínez, J. (2015). Problematización, eventualización y ficcionalización: la crítica en la visibilización de las subjetividades. Tabula Rasa, 22, 69-83.

Martínez-Posada, J. E., Hernández-Molina, N. y Hernández-Molina, L. M. (2019). Ontología crítica de las prácticas formativas en medicina y enfermería. Magis, Revista Internacional de Investigación en Educación, 11(23), 135-150. https://doi.org/10.11144/Javeriana.m1123.ocpf

Mejía, M. R. (2010). La sistematización. Empodera y produce saber y conocimiento. Bogotá: Desde Abajo.

Ministerio de Educación Nacional. (2016). Becas para la excelencia docente. http://gabo.mineducacion.gov.co/becasdocentes/que_es.aspx

Ministerio de Educación Nacional. (2018). Programa Todos a Aprender (PTA) se fortalece en 2019. https://www.mineducacion.gov.co/1759/ w3-article-379147.html?_noredirect=1

Nadeau, J. (2004). La practique comme lieu de la théologique practique. Laval théologie et philosophique, 2(60), 205-224.

Navarro, P. y Díaz, C. (1999). Análisis de contenido. En J. M. Delgado y J. Gutiérrez, Métodos y técnicas cualitativas de investigación en ciencias sociales (pp. 177-244). Madrid: Síntesis.

Organización de las Naciones Unidas para la Educación, la Ciencia y la Cultura, Unesco. (2014a). Se busca: docentes capacitados a fin de que todos los niños puedan ejercer su derecho a la enseñanza primaria. http://es.unesco.org/gem-report/se-busca-docentes-capacitados-fin-de-que-todos-los-ni\%C3\%B1 os-puedan-ejercer-su-derecho-la-ense\%C3\%B1anza\#sthash.c0L6PbeU.s1HjrEba.dpbs

Organización de las Naciones Unidas para la Educación, la Ciencia y la Cultura, Unesco. (2014b). Informe de seguimento de la EPT en 
el mundo 2013/4. Enseñanza y aprendizaje: lograr la calidad para todos. París: Autor.

Organización de las Naciones Unidas para la Educación, la Ciencia y la Cultura, Unesco. (2015). Informe de seguimiento de la EPT en el mundo. La educación para todos, 2010-2015: logros y desafíos. París: Autor.

Organización de las Naciones Unidas para la Educación, la Ciencia y la Cultura, Unesco. (2016). Informe de seguimiento de la educación en el mundo, 2016. La educación al servicio de los pueblos y el planeta: creación de futuros sostenibles para todos. París: Autor.

Organización de las Naciones Unidas para la Educación, la Ciencia y la Cultura, Unesco. (2017). Informe de seguimento de la educación en el mundo 2017/8. Rendir cuentas en el ámbito de la eduación. Cumplir nuestros compromisos. París: Autor.

Organización para la Cooperación y el Desarrollo Económico, OCDE. (2016). Educación en Colombia. Aspectos destacados. 2016. París: Autor.

Organización para la Cooperación y el Desarrollo Económico, OCDE. (2017). Estudios económicos de la OCDE. Bogotá: Autor.

Ortega, P. y Torres, A. (2011). Lola Cendales González, entre trayectos y proyectos en la educación popular. Revista Colombiana de Educación, 61, 333-357.

Perreau, L. (Ed.). (2010). L'expérience. París: Librairie philosophique J. Vrin.

Rodríguez, B. C. (1989). La educación popular en América Latina. Quito: Cedep.

Secretaría de Educación del Distrito de Bogotá. (2012). Plan Sectorial de Educación 2011-2018. http://www.educacionbogota.edu.co/archivOS/SECTOR_EDUCATIVO/PLAN_SECTORIAL/2013/Bases\%20Plan\%20Sectorial\%20Educacion\%20DEF\%2024072013.pdf.

Sime, L. (1991). Los discursos de la eduación popular. Lima: Tarea.

Torres, A. (1996). Enfoques cualitativos y participativos de investigación social. Bogotá: Unisur.

Torres, A. (1998). La sistematización de experiencias educativas: reflexiones sobre una práctica reciente. Pedagogía y Saberes, 13, 6-17.

Torres, A. (2012). La educación popular. Trayectoria y actualidad. Bogotá: El Búho.

Torres, A. (2013). El retorno a la comunidad. Bogotá: Cinde-El Búho.

Verger i Planells, A. (2007). Sistematizando experiencias: análisis y recreación de la acción colectiva desde la educación popular. Revista de Educación, 343, 623-645. 